\title{
Efficacy and Safety of Topical Calcipotriol $0.005 \%$ Versus Topical Clobetasol $0.05 \%$ in the Management of Alopecia Areata: An Intrasubject Pilot Study
}

\author{
Elisa Molinelli · Anna Campanati - Valerio Brisigotti • \\ Claudia Sapigni · Matteo Paolinelli · Annamaria Offidani
}

Received: March 11, 2020 / Published online: April 27, 2020

(C) The Author(s) 2020

\section{ABSTRACT}

Introduction: Alopecia areata (AA) is a chronic inflammatory non-scarring type of hair loss. Current therapies for alopecia areata are rather limited and mainly involve the use of topical, intra-lesional or systemic steroids and topical immunotherapy, with variable benefit. Recent studies have demonstrated that vitamin D analogues could potentially promote hair growth in patients with patchy AA.

Methods: We investigated the efficacy and safety of treatment with calcipotriol, a synthetic derivative of vitamin D (calcipotriol $0.005 \%$ ointment), versus treatment with the corticosteroid clobetasol (topical clobetasol $0.05 \%$ formulation), in a series of 35 patients with scalp $\mathrm{AA}$, using an intrasubject design.

Digital Features To view digital features for this article go to https://doi.org/10.6084/m9.figshare.12097728.

E. Molinelli $(\bowtie) \cdot$ A. Campanati · V. Brisigotti ·

C. Sapigni · M. Paolinelli · A. Offidani

Dermatological Unit, Department of Clinical and

Molecular Sciences, Polytechnic Marche University,

Conca 71 Street, Ancona, Italy

e-mail: molinelli.elisa@gmail.com
Results: Patches treated with calcipotriol ointment showed greater and faster response rates than did those treated with topical clobetasol, although the differences were not statistically significant. The main strength of the study is its prospective design; the main limitation is the small number of participants.

Conclusions: Treatment with the calcipotriol would appear to be reasonably effective in patients with mild to moderate patchy AA and was associated with only limited and reversible side effects.

Keywords: Alopecia areata; Calcipotriol; Clobetasol; Therapy; Treatment; Vitamin D analogue 


\section{Key Summary Points}

Alopecia areata (AA) is a chronic inflammatory non-scarring type of hair loss. Current therapies for alopecia areata are rather limited and mainly involve topical, intra-lesional or systemic steroids and topical immunotherapy, with variable benefit.

The clinical efficacy of topical vitamin D analogues in AA is still open to debate, although a recent study has demonstrated that the synthetic vitamin D3 analogue calcipotriol is an effective alternative treatment of AA of the scalp.

The aim of this study was to evaluate the efficacy and safety of a topical calcipotriol formulation versus a topical corticosteroid (clobetasol) formulation, in the treatment of limited patchy AA of the scalp.

Calcipotriol, which is characterized by a favourable side effects profile, seems to be a valid therapeutic option in the management of AA.

\section{INTRODUCTION}

Alopecia areata (AA) is a common, inflammatory, non-scarring type of hair loss that affects persons of both sexes and all age groups, with a prevalence in the general population of approximately $0.1-0.2 \%$. It is characterized by variable clinical presentations, ranging from single or multiple well-circumscribed patches of hair loss to extensive involvement with complete absence of body and scalp hair [1]. The condition is associated with a variety of inflammatory and immune-mediated diseases, such as atopic dermatitis, allergic rhinitis, lupus erythematosus, psoriasis, thyroid disease, rheumatoid arthritis, celiac disease and type 1 diabetes mellitus [2].

Several therapies are available for the treatment of AA, including corticosteroids (topical, systemic or injectable modalities) and topical immunotherapies (diphenylcyclopropenone or squaric acid dibutylester), but these are characterized by unpredictable efficacy and durability $[3,4]$. It has been demonstrated that 1,25-dihydroxyvitamin $\mathrm{D}(3)$ receptors (VDRs) are strongly expressed in the keratinocytes of human and murine hair follicles and that the lack of expression of VDRs is associated with reduced hair follicle growth and epidermal differentiation. Studies of the scalps of AA patients have also shown reduced VDR expression in the hair follicles of affected areas [5].

Results from recent studies suggest that topical application of the vitamin D3 analogue calcipotriol has a beneficial effect on patchy AA of the scalp $[5,6]$. Given this background, we have evaluated the efficacy and safety profile of the topical calcipotriol $0.005 \%$ ointment versus a topical corticosteroid ointment (clobetasol $0.05 \%$ ) in a series of 35 patients with the diagnosis of AA of the scalp.

\section{METHODS}

The study population comprised 35 patients with patchy, mild-to moderate AA of the scalp. The diagnosis of AA was made based on the typical clinical presentation (round or oval patches of non-scarring hair loss) and characteristic dermoscopic findings (yellow dots, short regrowing hairs and exclamation mark hairs). Inclusion criteria were: male and female patients aged > 16 years; diagnosis of AA; 12 weeks of wash-out from previous treatments. Age, sex, onset, duration and progress of the disease, precipitating factors, family history of alopecia or other autoimmune disorders and history of previous treatments taken were recorded in a digital platform. Investigations to rule out anaemia, diabetes and thyroid dysfunction were performed at baseline. Each patient was evaluated for the site, number and size of alopecic lesions, following which we calculated the extent of involvement in terms of overall alopecic area (expressed in $\mathrm{cm}^{2}$; average surface of the alopecic area ranged from 1.5 to $3 \mathrm{~cm}^{2}$ ) in the two hemispheres (right and left side), respectively. Patients with the same 
extent of patchy AA in the two hemispheres were specifically recruited in order allow comparison of the treatment of alopecic areas in an intrasubject study design. Calcipotriol $0.005 \%$ ointment $(50 \mu \mathrm{g}$ calcipotriol monohydrate $/ \mathrm{mL})$ and clobetasol propionate $0.05 \%$ ointment were applied twice daily for 12 weeks using the intrapatient design (right vs. left side). A total of 46 and 41 patchy AA areas were treated with calcipotriol $0.005 \%$ ointment and clobetasol propionate $0.05 \%$ ointment, respectively. From week 13 to week 24 , all patients decided to continue with the treatment at the same dosage schedule. The effectiveness of the treatment was evaluated by clinical interview and physical examination after 12 and 24 weeks. Photographic documentation was obtained at baseline, after 12 weeks of treatment and every 12 weeks thereafter, up to 12 months.

The alopecia grading score (AGS) was calculated at baseline and after 12 and 24 weeks of treatment using a $0-5$ score $(0=$ no alopecia; $1=$ hair loss $<10 \% ; 2=$ hair loss $11-25 \%$; $3=$ hair loss $26-50 \%$; $4=$ hair loss $51-75 \%$; $5=$ hair loss $>75 \%)$. The primary outcome of the trial was to evaluate the hair regrowth rate, assessed using a 5-point semiquantitative regrowth score $(\mathrm{RGS}) \quad(0=$ regrowth $<10 \%$; $1=$ regrowth $11-25 \% ; 2=$ regrowth $26-50 \%$; $3=$ regrowth $51-75 \% ; 4=$ regrowth $>75 \%$ ) at 12 weeks. Cosmetically acceptable regrowth was defined as hair growth sufficient to cover the alopecic area and thereby concealing the area. Complete hair regrowth was considered to be hair regrowth of $\geq 90 \%$. Patients were followed up for 6 months after the end of treatment to evaluate the persistence of response/time to relapse. Relapse was considered to be a 10\% worsening in terms of hair loss in comparison to the previous evaluation, both during and after treatment.

Statistical analysis was performed using GraphPad Prism software (version 7.0; GraphPad Software, Inc., San Diego, CA, USA). The differences between baseline values and those after treatment were compared using a Wilcoxon matched-pairs test. Differences between placebo and active group values were analysed using the Mann-Whitney $U$ test. All performed tests were two-tailed. The paired Student's $t$ test was utilized to compare continuous variables. Continuous variables were expressed as means \pm standard deviation (SD), and ordinal variables were presented as mean, median and range. A $p$ value of $<0.05$ was considered to be statistically significant.

The study conformed with the Helsinki Declaration of 1964, as revised in 2013, concerning human and animal rights. Ethics committee approval was not required as all patients were treated with effective topical therapies (the use of AA calcipotriol appears to be supported by data in the literature) and calcipotriol is already successfully used in our clinical practice in the treatment of AA of the beard. Informed consent was obtained for each participant.

\section{RESULTS}

The study included 35 Caucasian patients (19 men, 16 women) with a mean age of 32.3 ( \pm SD 8.1; range 19-56) years. All patients were

Table 1 Demographic and clinical characteristics of the study population with alopecia areata

\begin{tabular}{lr}
\hline $\begin{array}{l}\text { Demographic and clinical } \\
\text { characteristics }\end{array}$ & \multicolumn{1}{l}{ Values } \\
\hline Gender, $n$ (\%) & $19(54.29)$ \\
Male & $16(45.71)$ \\
Female & $32.34(8.14)$ \\
Age, mean (SD) & $27.11(10.01)$ \\
Onset age, mean (SD) & $27(77.14)$ \\
Family history of AA, $n$ (\%) & $8(22.86)$ \\
No & $12(34.29)$ \\
Yes & $12(34.29)$ \\
Alopecia grading score (AGS) T0, $n(\%)^{a}$ & $11(31.43)$ \\
2 & \\
3 & \\
4 &
\end{tabular}


Table 2 Regrowth score after 12 or 24 weeks of treatment

\begin{tabular}{|c|c|c|c|c|c|c|c|c|}
\hline \multirow{2}{*}{$\begin{array}{l}\text { Regrowth score } \\
\text { (RGS) }^{\mathbf{a}}\end{array}$} & \multicolumn{4}{|l|}{12 weeks } & \multicolumn{4}{|l|}{24 weeks } \\
\hline & Total & Calcipotriol & Clobetasol & $p$ & Total & Calcipotriol & Clobetasol & $p$ \\
\hline 1 & $20(28.57)$ & $7(20.00)$ & $13(37.14)$ & 0.407 & $13(18.57)$ & $4(11.43)$ & $8(22.86)$ & 0.814 \\
\hline 2 & $4(5.71)$ & $2(5.71)$ & $2(5.71)$ & & $3(4.29)$ & $2(5.71)$ & $1(2.86)$ & \\
\hline 3 & $8(11.43)$ & $4(11.43)$ & $4(11.43)$ & & $9(12.86)$ & $5(14.29)$ & $4(11.43)$ & \\
\hline 4 & $38(54.29)$ & $22(62.86)$ & $16(45.71)$ & & $45(64.29)$ & $24(68.77)$ & $22(62.86)$ & \\
\hline
\end{tabular}

Values in table are given as the number with the percentage in parenthesis

${ }^{\text {a }}$ RGS: $0=$ regrowth $<10 \% ; 1=$ regrowth $11-25 \% ; 2=$ regrowth $26-50 \% ; 3=$ regrowth $51-75 \%$; $4=$ regrowth $>75 \%$

Table 3 Relapse

\begin{tabular}{llccl}
\hline Relapse & Total & Calcipotriol & Clobetasol & $\boldsymbol{p}$ \\
\hline No & $60(85.71)$ & $32(91.43)$ & $28(80.00)$ & 0.306 \\
Yes & $10(14.29)$ & $3(8.57)$ & $7(20.00)$ & \\
\hline
\end{tabular}

Values in table are given as the number of calcipotrioltreated patches and clobetasol-treated sites, respectively, with the percentage in parenthesis

affected by mild to moderate AA at baseline (AGS 2-4). The mean age of onset of AA was 27.1 ( \pm SD 10.01; range 11-53) years. Eight patients $(22.9 \%)$ were found to have a family history of any type of AA (Table 1). No symptoms were detected in any case. All treated patients completed the study period of 12 weeks and were included in the final analysis after 24 weeks. All patients applied calcipotriol $0.005 \%$ ointment twice daily for 12 weeks on target AA areas and clobetasol propionate $0.05 \%$ twice daily for 12 weeks on contralateral control AA areas (right vs. left).

At week 12, hair regrowth of $>75 \%$ (RGS $=$ 4) was observed at $62.9 \%$ of the scalp sites (22/ 35 ) treated with the vitamin $D$ analogue calcipotriol and $45.7 \%$ of scalp sites (16/35) treated with the topical corticosteroid clobetasol. Hair regrowth of $>25 \%(\mathrm{RGS}=2)$ was observed at $5.7 \%$ of scalp sites $(2 / 35)$ treated with calcipotriol and also at $25 \%$ of scalp sites $(2 / 35)$ treated with clobetasol. Hair regrowth of $\geq 50 \%$ (RGS $=3$ ) was observed at $11.4 \%$ of calcipotrioltreated scalp sites $(4 / 35)$ and also at $11.4 \%$ of clobetasol propionate-treated scalp sites $(11.4 \%)$. At 12 weeks, complete hair regrowth (hair regrowth of $\geq 90 \%$ ) was observed in six of the 22 patients achieving hair regrowth of $>$ $75 \%(\mathrm{RGS}=4)$ at calcipotriol-treated sites and in two of the 16 patients achieving hair regrowth of $>75 \% \quad(\mathrm{RGS}=4)$ at clobetasoltreated sites. From weeks 13 to 24 , all patients continued with the treatment at the same dosage schedule. At week 24, 69\% of calcipotriol-treated areas and $63 \%$ of clobetasoltreated areas were assessed with RGS $=4$, indicating complete hair regrowth. Telangiectasia and moderate pruritus were observed in three patients, respectively, both whose AA areas were treated with clobetasol propionate. Two patients experienced mild erythema and pruritus on patches treated with calcipotriol $0.005 \%$ ointment. During the 12 months of follow-up after the end of the treatment, relapses were observed in three and seven calcipotriol-treated patches and clobetasol-treated sites, respectively. Evolution and extent of regrowth (RGS) in calcipotriol- and clobetasol-treated lesions at weeks 12 and 24 are summarized in Tables 2 and 3.

\section{DISCUSSION}

Alopecia areata is a T-cell-mediated autoimmune disease that leads to non-scarring alopecia. The pathogenesis of AA remains incompletely understood to date, although a complex interplay between loss of immune 
privilege in the hair follicle, autoimmune-mediated hair follicle destruction and upregulation of inflammatory pathways has been advocated to explain the development of this condition. Autoreactive CD8 and CD4 T cells infiltrate the hair follicles and attack hair follicle-derived autoantigens while sparing the stem cell compartment [7].

Corticosteroids are widely used for the treatment of patchy AA, and intralesional steroids are considered to be a first-line treatment for patchy hair loss of limited extent [8]. However repeated injections of corticosteroids are associated with undesirable side effects, including skin atrophy, and injectable corticosteroids should be used with caution in the pediatric population. Topically applied steroids, in particular clobetasol propionate $0.05 \%$, which is a superpotent corticosteroid, are widely used as monotherapy for patchy limited AA, both in adult and pediatric patients. However, acneiform eruption, telangiectasia and skin atrophy can commonly complicate the topical use of corticosteroids $[8,9]$.

Only a few studies have described the efficacy of topical vitamin $\mathrm{D}$ analogues in the treatment of AA $[5,6,10]$. Vitamin D3 derivatives have been shown to have several biologically active effects, including the regulation of epidermal cell proliferation and differentiation and modulation of cytokine production. 1,25Dihydroxyvitamin D inhibits Th1 cytokine secretion and stimulates Th2 cytokine production $[11,12]$. The shift from the Th1 phenotype to the Th2 phenotype suppresses Th1-mediated autoimmune diseases, as has already been demonstrated in psoriasis $[13,14]$. In addition, 1,25-dihydroxyvitamin $\mathrm{D}$ inhibits the function of Th17 cells, potent inducers of autoimmune diseases, and enhances regulatory $\mathrm{T}$ cells, which play a crucial role in suppressing autoimmune responses [15].

The results of our study indicate that both the topical vitamin D analogue and topical corticosteroid formulations were safe and effective therapies for treating scalp AA (Table 2). Although the differences were not statistically significant, calcipotriol was associated with faster hair regrowth and a higher percentage of patches with complete response versus clobetasol. In addition, calcipotriol-treated patches maintained the benefit longer than clobetasol-treated lesions (Table 3). Patients treated with topical corticosteroids showed a greater and faster tendency to relapse (20 weeks) versus patients treated with the topical vitamin D analogue (48 weeks).

Our study highlights the following advantages of the calcipotriol formulation over the topical steroids: easy availability, affordability, efficacy in treating AA and minimal and reversible side effects.

The main limitations of this study are the small sample size and the lack of evaluation over the long term. Thus, further studies with large cohort, well-selected populations and a clinically relevant endpoint such as long-term overall regrowth are essential to corroborate these findings.

\section{CONCLUSION}

In conclusion, we found evidence to support the efficacy of the topical vitamin $\mathrm{D}$ analogue formulation for the treatment of limited AA, with a lower incidence of adverse events compared with the topical steroid formulation. Randomized controlled trials are required to confirm these results. In addition, further research is needed to assess the efficacy and safety profile of this vitamin $D$ analogue in treating pediatric AA and the efficacy of a combination therapy of topical steroids and calcipotriol.

\section{ACKNOWLEDGEMENTS}

The authors would like to thank Dr. Mirko Di Rosa, who provided statistical analysis assistance in the preparation of this article, and the participants of the study

Funding. No funding or sponsorship was received for this study or publication of this article.

Authorship. All named authors meet the International Committee of Medical Journal 
Editors (ICMJE) criteria for authorship for this article, take responsibility for the integrity of the work as a whole, and have given their approval for this version to be published.

Disclosures. Elisa Molinelli, Anna Campanati, Valerio Brisigotti, Claudia Sapigni, Matteo Paolinelli, Annamaria Offidani have nothing to disclose.

Compliance with Ethics Guidelines. The study conformed with the Helsinki Declaration of 1964, as revised in 2013, concerning human and animal rights. Ethics committee approval was not required as all patients were treated with effective topical therapies (the use of AA calcipotriol appears to be supported by data in the literature) and calcipotriol is already successfully used in our clinical practice in the treatment of AA of the beard. Informed consent was obtained for each participant.

Data Availability. The datasets analysed during the current study are available from the corresponding author on reasonable request.

Open Access. This article is licensed under a Creative Commons Attribution-NonCommercial 4.0 International License, which permits any non-commercial use, sharing, adaptation, distribution and reproduction in any medium or format, as long as you give appropriate credit to the original author(s) and the source, provide a link to the Creative Commons licence, and indicate if changes were made. The images or other third party material in this article are included in the article's Creative Commons licence, unless indicated otherwise in a credit line to the material. If material is not included in the article's Creative Commons licence and your intended use is not permitted by statutory regulation or exceeds the permitted use, you will need to obtain permission directly from the copyright holder. To view a copy of this licence, visit http://creativecommons.org/licenses/bync/4.0/.

\section{REFERENCES}

1. Strazzulla LC, Wang EHC, Avila L, et al. Alopecia areata: disease characteristics, clinical evaluation, and new perspectives on pathogenesis. J Am Acad Dermatol. 2018;78:1-12.

2. Ganzetti G, Campanati A, Offidani A. Alopecia areata: a possible extraintestinal manifestation of Crohn's disease. J Crohns Colitis. 2012;6:962-3.

3. Lee S, Lee WS. Management of alopecia areata: updates and algorithmic approach. J Dermatol. 2017;44:1199-211.

4. Ganzetti G, Campanati A, Simonetti O, Cataldi I, Giuliodori K, Offidani A. Videocapillaroscopic pattern of alopecia areata before and after diphenylciclopropenone treatment. Int J Immunopathol Pharmacol. 2008;24:1087-91.

5. Çerman AA, Solak SS, Altunay İ, Küçükünal NA. Topical calcipotriol therapy for mild-to-moderate alopecia areata: a retrospective study. J Drugs Dermatol. 2015;14:616-20.

6. Narang T, Daroach M, Kumaran MS. Efficacy and safety of topical calcipotriol in management of alopecia areata: a pilot study. Dermatol Ther. 2017;30(3):e12464. https://doi.org/10.1111/dth. 12464.

7. Rajabi F, Drake LA, Senna MM, Rezaei N. Alopecia areata: a review of disease pathogenesis. Br J Dermatol. 2018;179:1033-48.

8. Messenger AG, McKillop J, Farrant P, McDonagh AJ, Sladden M. British Association of Dermatologists' guidelines for the management of alopecia areata. Br J Dermatol. 2012;166:916-26.

9. Strazzulla LC, Wang EHC, Avila L, et al. Alopecia areata: an appraisal of new treatment approaches and overview of current therapies. J Am Acad Dermatol Venereol. 2018;78:15-24.

10. Kim DH, Lee JW, Kim IS, et al. Successful treatment of alopecia areata with topical calcipotriol. Ann Dermatol. 2012;24:341-4.

11. Di Rosa M, Malaguarnera M, Nicoletti F, et al. Vitamin D3: a helpful immuno-modulator. Immunology. 2011;134:123-39.

12. Hewison M. An update on vitamin D and human immunity. Clin Endocrinol. 2012;76:315-25.

13. Ganzetti G, Campanati A, Scocco V, et al. The potential effect of the tumour necrosis factor-acinhibitors on vitamin D status in psoriatic patients. Acta Derm Venereol. 2014;94:715-7. 
14. Daroach M, Narang T, Saikia UN, Sachdeva N, Sendhil KM. Correlation of vitamin D and vitamin $\mathrm{D}$ receptor expression in patients with alopecia areata: a clinical paradigm. Int J Dermatol. 2018;57: 217-22.
15. Lee $\mathrm{S}$, Kim BJ, Lee $\mathrm{CH}$, Lee WS. Increased prevalence of vitamin D deficiency in patients with alopecia areata: a systematic review and meta-analysis. J Eur Acad Dermatol Venereol. 2018;32:1214-21. 